\title{
Thermodynamics for the Zero-Level Set of the Brownian Bridge ${ }^{\star}$
}

\author{
Dimitri Petritis $\star \star$ \\ Institut de Physique Théorique, Université de Lausanne, CH-1015 Lausanne, Switzerland
}

\begin{abstract}
The random set of instants where the Brownian bridge vanishes is constructed in terms of a random branching process. The Hausdorff measure supported by this set is shown to be equivalent to the partition function of a special class of disordered systems. This similarity is used to show rigorously the existence of a phase transition for this particular class of disordered systems. Moreover, it is shown that at high temperature the specific free energy has the strong self-averaging property and that at low temperature it has no selfaveraging property. The unicity at high-temperature and the existence of many limits at low temperature are established almost surely in the disorder.
\end{abstract}

\section{Introduction}

Random walks are thoroughly studied in many areas of physics and applied mathematics. A challenging problem that remains open is the construction of the measure for the self-avoiding walk in low dimension (i.e. $d=2$ or 3). A promising method towards that aim is the study of weakly self-avoiding random walks by suppressing walks with many self-intersections $[27,28]$. Counting the selfintersections of a walk led to many new concepts; the most useful one seems to be that of local time. Intuitively, if $X_{s}, s \in[0, \infty$ [ is a random process with values in $\mathbf{R}$, its local time on $x \in \mathbf{R}$ can be defined formally as

$$
L_{t}(x)=\int_{0}^{t} \delta\left(X_{s}-x\right) d s
$$

For Brownian motion, this concept can be given a rigorous meaning [20] and it turned out that it is intimately connected to the Hausdorff measure of the $x$-level sets of the Wiener process, i.e. of the random sets of instants where the Brownian motion attains the value $x$ [15]. The study of the weakly self-avoiding walks and their intersections was the starting point of this work [17].

\footnotetext{
* Work supported by the Swiss National Science Foundation

$\star \star$ Permanent address: Institut mathématique, Université de Rennes I, Campus de Beaulieu, F-35042 Rennes Cedex, France
} 
Another interesting problem - apparently completely disconnected from random walks - is the statistical mechanics of disordered systems, like the random field Ising model, spin glasses etc. These physical systems are very difficult to study from a rigorous point of view and only few partial results are known [12,4,25]. Since a general mathematical setting for these systems is still lacking, it seems interesting to study some simplified models, sharing the main features of the "real" systems, in order to grasp the mathematical difficulties. The purpose of this paper is to present such a model that arose in the study of the astonishingly different problem of the level sets of the Brownian bridge.

The problem encountered in the statistical mechanics of disordered systems is very simple to explain in principle. However, nobody knows the complete solution up to now. To start, one needs some finite-volume configuration space $X$ (e.g. $X=\{-1,1\}^{[-n, n] \cap \mathbf{Z}}$ is the configuration space for the finite-volume, lattice, onedimensional Ising systems). This space is turned into a measure space by equipping its Borel $\sigma$-field, $\mathscr{B}(X)$, with an a priori measure $\kappa$. Very often (for discrete finite systems) $\kappa$ is chosen to be the counting measure. The disorder is modelised by some underlying probability space $(\Omega, \mathscr{F}, \rho)$. Assume that some positive measurable function $f: \Omega \times X \rightarrow \mathbf{R}^{+}$is defined and construct for each $\omega \in \Omega$ the measure

$$
\mu_{\omega, \beta}(B)=\int_{B}[f(\omega, x)]^{\beta} \kappa(d x)
$$

for $\beta>0$ and $B \in \mathscr{B}(X)$. In the standard terminology, $f$ is the exponential of the Hamiltonian and $\beta$ is interpreted as the inverse temperature. $\mu$ is a random measure on $(X, \mathscr{B}(X))$, i.e. a measure-valued random variable. The total mass $\mu_{\omega, \beta}(X)$ is known as the finite-volume partition function $Z_{\omega}(\beta)$; obviously, in the disordered case it is a random variable. As far as the volume is finite, $(X, \mathscr{B}(X))$ can be equipped with a natural random probability measure defined by

$$
\pi_{\omega, \beta}=Z_{\omega}(\beta)^{-1} \mu_{\omega, \beta} .
$$

The problem, as is usual in statistical mechanics, lies in the infinite volume limit (thermodynamic limit): the partition function may vanish in that case for certain values of the inverse temperature $\beta$ and $\pi_{\omega, \beta}$ may be not uniquely defined. In summarising, one has to study whether, for some $\beta$, there exists a unique probability measure defined as the thermodynamic limit of $\pi_{\omega, \beta}$ or there are many subsequences converging to different limits. The complete answer is given for the particular disordered system considered here. The paper is organised as follows:

In Sect. 2, the definition of the zero-level set of the Brownian bridge and a random recursive construction of this set are given. In Sect. 3 the random Hausdorff measure of the zero-level set is shown to be equal to the partition function of a disordered system of the Derrida's GREM-type. Standard martingale arguments are used to prove the vanishing of the partition function at low temperature.

The main results are postponed to Sect. 4. Namely, it is shown in that section that at high temperature there is a unique probability measure and at low temperature there are many states. Moreover, the existence of a specific free energy is proved at every temperature with a self-averaging property at high temperature and lack of self-averaging at low temperature. Section 5 is devoted to the study 
of the critical point. A characterisation of the probability measure is given in terms of the exact Hausdorff measure of the zero-level set of the Brownian bridge. Some open problems are discussed in Sect. 6.

In [16], rigorous probabilistic methods are used to study the phase transition of a REM-type model; for GREM-type models, rigorous results, reminiscent of the ones presented here, are obtained for the first time for a model of random multiplications on the $c$-adic tree by Collet and Koukiou [7]. Some of their powerful methods are used here for the study of the high temperature region, different methods are used for the low temperature region.

Although the notation used is standard and defined before it is used, it is useful to remember that, in general, small Greek letters are used to denote measures; especially $\lambda$ is always the Lebesgue measure and $\pi$ and $\rho$ are always probability measures. Script capital Latin letters are used for $\sigma$-fields; exceptionally, the symbol $\mathscr{H}$ is used to denote the Hausdorff outer measure and not a $\sigma$-field.

\section{The Zero-Level Set of the Brownian Bridge}

2.1. Definition of the Zero-Level Set. A one-dimensional Brownian bridge over $[0,1]$ is a centered, Gaussian random process $B_{t}(\omega), t \in[0,1]$ over a probability space $(\Omega, \mathscr{F}, \rho)$ and taking values in $\mathbf{R}$, with covariance function $E\left(B_{s} B_{t}\right)=s(1-t)$ for $0 \leqq s \leqq t \leqq 1$. Obviously, $B_{0}=B_{1}=0$. We are interested in the level sets of $B_{t}$, i.e. the random sets of constant $B$. More specifically we have the following

Definition 2.1. The random set

$$
z_{\omega}=\left\{t \in[0,1]: B_{t}(\omega)=0\right\}
$$

is called the zero-level set of the Brownian bridge.

This set has a Cantor-like structure as stated in the following

Lemma 2.2. The zero-level set of the Brownian bridge almost surely

i) has Lebesgue measure zero

ii) is closed

iii) has accumulation points at $t=0$ and $t=1$

iv) has no isolated points in $] 0,1[$.

Proof. The proof of items i)-iii) follows closely the one given in [15] for the Wiener process; it is therefore only sketched in the present situation. Denote by $\lambda$ the Lebesgue measure over $\mathscr{B}(\mathbf{R})$.

i) By Fubini's theorem

$$
E\left(\lambda\left(z_{\omega}\right)\right)=\int_{0}^{1} d t \rho\left(\left\{B_{t}=0\right\}\right)=0
$$

hence $\lambda\left(z_{\omega}\right)=0$ a.s.

ii) There exists almost surely a version of the random mapping $t \mapsto B_{t}(\omega)$ which is continuous and $z_{\omega}$ is the inverse image under this a.s. continuous mapping of the closed set $\{0\}$; it is therefore closed. 
iii) It is enough to show that $\forall \varepsilon \in] 0,1\left[\right.$, the process $B_{t}$ changes sign infinitely often for $t \in[0, \varepsilon]$. For every $C \in \mathscr{B}(\mathbf{R})$, consider the hitting time $H_{C}(\omega)$ of this set, i.e. $H_{C}(\omega)=\inf \left\{t \in[0,1]: B_{t}(\omega) \in C\right\}$. One can show that both $H_{] 0, \infty[}$ and $H_{]-\infty, 0[}$ are optional. Moreover, because of the symmetry around the origin of the Brownian bridge we have $\rho\left(H_{\mathrm{J}, \infty[}=0\right)=\rho\left(H_{\mathrm{]}-\infty, 0 \mathrm{[}}=0\right)=p$. By the Blumenthal zero-one law, $p$ can be either 0 or 1 . Now, $p$ can not be 0 since this would mean that there is an interval $[0, \zeta]$, with $\zeta>0$ such that $B_{t}=0$ for every $t \in[0, \zeta]$ almost surely; this issue is impossible. So, $p=1$ and one can find sequences of times $s_{n}, t_{n} \downarrow 0$ such that $B_{s_{n}}<0$ and $B_{t_{n}}>0$ a.s. for $n>1$. Thus 0 is an accumulation point. By symmetry of the probability distribution, with respect to reflexion on $t=1 / 2$, the point 1 is also an accumulation point.

iv) For every $t \in] 0,1[$ define the random times straddling $t$ by

and

$$
\left.\left.\beta_{t}(\omega)=\inf \{s \in] t, 1\right]: B_{s}(\omega)=0\right\}
$$

$$
\gamma_{t}(\omega)=\sup \left\{s \in \left[0, t\left[: B_{s}(\omega)=0\right\} .\right.\right.
$$

Suppose that some optional time $T \in] 0,1[$ is an isolated zero of the Brownian bridge and consider $\beta_{T}(\omega)$ and $\gamma_{T}(\omega)$. The set $z_{\omega}$ being closed by item ii) above, $B_{t}$ is still a Brownian bridge over both $\left[\gamma_{T}(\omega), T\right]$ and $\left[T, \beta_{T}(\omega)\right]$. By shifting and rescaling of the bridges and item iii) above, it follows that $t=T$ is an accumulation point, in contradiction to the hypothesis that it was an isolated point. It remains to show that $T$ is always an optional time, but this is immediate since $T=\inf \left\{s \geqq T: B_{s}=0\right\}$.

The previous lemma establishes that $z_{\omega}$ is not a trivial set. Although it has zero Lebesgue measure, it is closed and dense in itself. To get some insight into the structure of this set it is natural to look at its Hausdorff dimension. Some basic facts about Hausdorff measures are reminded in the next subsection.

2.2. The Hausdorff Dimension. The Hausdorff dimension [13] allows one to distinguish between sets of same Lebesgue measure. It can be defined on every metric space $(R, d)$ and has interesting mathematical properties [3] (see also [10] for a modern exposition).

Definition 2.3. Let $A$ be a subset of a metric space $(R, d)$. A countable collection $\left\{U_{i}\right\}, i \in \mathbf{N}$ of subsets of $R$ is called a $\delta$-cover of $A$ if

i) $A \subset \bigcup_{i \in \mathbf{N}} U_{i}$

ii) $\left|U_{i}\right|<\delta, \forall i$, where $\left|U_{i}\right| \equiv \sup \left\{d(x, y): x, y \in U_{i}\right\}$.

Given some non-negative number $s$ and some subset $A \subset R$, define

$$
\mathscr{H}_{\delta}^{s}(A)=\inf \sum_{i=1}^{\infty}\left|U_{i}\right|^{s},
$$

where the infimum is over all $\delta$-covers of $A$. Now, define an exterior measure by taking

$$
\mathscr{H}^{s}(A)=\lim _{\delta \downarrow 0} \mathscr{H}_{\delta}^{s}(A) .
$$


Notice that $\mathscr{H}_{\delta}^{s}(A)$ does not decrease when $\delta$ decreases, therefore the above limit always exists (may be infinite). $\mathscr{H}^{s}(A)$ is the Hausdorff measure of order $s$ as originally defined by Hausdorff. In the special case of the metric space $R=\mathbf{R}$ with distance $d(x, y)=|x-y|$ the cover can be formed by intervals and the outer Hausdorff measure can be written as

$$
\mathscr{H}^{s}(A)=\lim _{\delta \downarrow 0}\left(\inf \sum_{i=1}^{\infty} \lambda\left(U_{i}\right)^{s}\right),
$$

where $\lambda\left(U_{i}\right)$ is the Lebesgue measure of the interval $U_{i}$.

Definition 2.4. The Hausdorff dimension, $d_{H}(A)$, of the set $A$ is given by

$$
d_{H}(A) \equiv \inf \left\{s: \mathscr{H}^{s}(A)=0\right\}=\sup \left\{s: \mathscr{H}^{s}(A)=\infty\right\} .
$$

Remark. $d_{H}(A)$ is the unique real number for which $\mathscr{H}^{s}(A)=\infty$ holds if $s<d_{H}(A)$ and $\mathscr{H}^{s}(A)=0$ if $s>d_{H}(A)$. Notice however that $\mathscr{H}^{d_{H}(A)}(A)$ may be zero, finite or infinite depending on the fine structure of the set $A$.

In view of the previous remark one may wonder whether it is possible to define some modified Hausdorff measure such that the exterior measure of $A$ of order $d_{H}(A)$ would be finite and non-zero, for the specific set $A$. Suppose that $A$ is a subset of $\mathbf{R}^{n}$. If $d_{H}(A) \neq n$, the set $A$ has zero $n$-dimensional Lebesgue measure. The Hausdorff measure rescales in some sense the Lebesgue measure to render it different from zero. However, the rescaling introduced by $\left|U_{i}\right|^{s}$ is quite crude and the corresponding Hausdorff measure is usually either 0 or $\infty$. Now, consider the function $h: \mathbf{R}^{+} \rightarrow \mathbf{R}^{+}$that can be written as $h(x)=x^{s} S(x), S(x)$ being a slowly varying function, i.e. a function with $\lim x^{\varepsilon} S(x)=0$ for every $\varepsilon>0$. Form the exterior measure $x \downarrow 0$

$$
\mathscr{H}^{(h)}(A)=\lim _{\delta \downarrow 0}\left(\inf \sum_{i=1}^{\infty} h\left(\left|U_{i}\right|\right)\right)
$$

where $h(x)=x^{s} S(x)$ as above. This definition generalises the notion of the Hausdorff measure, obviously it coincides with it if $S=1$. If there is some $s_{0}$ and some slowly varying function $S_{0}$ (i.e. $h(x)=x^{s_{0}} S_{0}(x)$ ) such that $0<\mathscr{H}^{(h)}(A)<\infty$, then $s_{0}$ is called the exact Hausdorff dimension of the set $A$.

The knowledge of the exact Hausdorff dimension of a set provides more detailed information about the set than the ordinary Hausdorff dimension. In fact, it gives not only the leading scaling term $\left(\left|U_{i}\right|^{d_{H}}\right)$ required to render the Lebesgue measure non-zero but also the subleading term included in $S_{0}(x)$.

In general, it is very difficult to compute the Hausdorff measure of a set just by applying the general Definition 2.4 since it is not a constructive one. In the following, a recursive random construction of the set $z_{\omega}$ is given that allows the computation of its Hausdorff measure by an appropriate limiting procedure.

\subsection{The Random Recursive Construction. Denote by $M=\{1,2\}$ and by $M^{*}=$ $\bigcup_{m=0}^{\infty} M^{m}$. Every element $s \in M^{*}$ is in an one-to-one correspondence with a branch of the rooted dyadic tree in an obvious way. Therefore, branches of the dyadic}


tree are also denoted by $s$. Denote by $|s|$ the integer-valued length of the branch $s$ and by $\left.s\right|_{n}, n \leqq|s|$ the restriction of $s$ to the $n$ first ancestors. Given two branches $s, t \in M^{*}$, we denote by $s \vee t$ the concatenation of $t$ to $s$ with length $|s \vee t|=|s|+|t|$. Notice that $s \vee t \neq t \vee s$ in general. By abuse of notation, write $s \vee 1$ (respectively $s \vee 2$ ) for the concatenation of branch (1) (respectively (2)) to $s$. Finally, use the symbol ( ) to denote the empty branch of length zero.

Now, given the stochastic process of the Brownian bridge $B_{t}$ over $(\Omega, \mathscr{F}, \rho)$ we construct a family of random, finite, closed intervals $\left\{K_{s}(\omega)\right\}, s \in M^{*}$, having the infinite, rooted, dyadic tree as index set. Denote by $a_{s}(\omega)$ and $b_{s}(\omega)$ the random edges of these intervals, i.e. $K_{s}(\omega)=\left[a_{s}(\omega), b_{s}(\omega)\right]$ and by $m_{s}(\omega)=\frac{1}{2}\left(a_{s}(\omega)+b_{s}(\omega)\right)$ their middle points. The recursive construction goes as follows:

Start from $K_{()}(\omega)=[0,1]=K$.

Define

$$
\begin{aligned}
& a_{(1)}=a_{()}=0, \\
& a_{(2)}=\inf \left\{t \geqq m_{()}: B_{t}(\omega)=0\right\}, \\
& b_{(1)}=\sup \left\{t \leqq m_{()}: B_{t}(\omega)=0\right\}, \\
& b_{(2)}=b_{()}=1,
\end{aligned}
$$

i.e. $K_{(1)}=\left[a_{(1)}, b_{(1)}\right]$ and $K_{(2)}=\left[a_{(2)}, b_{(2)}\right]$. The set $z_{\omega}$ being closed by Lemma 2.2, $B_{t}$ is by construction a Brownian bridge both over $K_{(1)}$ and $K_{(2)}$. Thus,

Repeat ad infinitum. Rescale and define in general

$$
\begin{aligned}
& a_{s \vee 1}=a_{s}, \\
& a_{s \vee 2}=\inf \left\{t \geqq m_{s}: B_{t}(\omega)=0\right\}, \\
& b_{s \vee 1}=\sup \left\{t \leqq m_{s}: B_{t}(\omega)=0\right\}, \\
& b_{s \vee 2}=b_{s} .
\end{aligned}
$$

Obviously, we have for the zero-level set of the Brownian bridge

$$
z_{\omega}=\bigcap_{m=1}^{\infty} \bigcup_{s \in M^{m}} K_{s}(\omega)
$$

The family, $\left\{D_{s}\right\}$, of deterministic intervals indexed by the dyadic tree is also used in the sequel. For every $s \in M^{k}$ there is exactly one interval $D_{s}$, of length $2^{-k}$, and belonging to the family $\left\{D_{s}\right\}$ that contains $K_{s}$.

Now it is possible to express the Hausdorff measure of $z_{\omega}$ in a form convenient for computations by virtue of the following

Lemma 2.5. Let

$$
Z_{\omega, k}(\beta)=\sum_{s \in M^{k}}\left(\lambda\left(K_{s}(\omega)\right)\right)^{\beta}
$$

with $\lambda$ denoting the Lebesgue measure. Then, the limit $\lim _{k \uparrow \infty} Z_{\omega, k}(\beta)=Z_{\omega}(\beta)$ always exists in $\mathbf{R} \cup\{+\infty\}$ and is equal to the Hausdorff measure of order $\beta$ of the zero-level set $z_{\omega}$, i.e. $Z_{\omega}(\beta)=\mathscr{H}^{\beta}\left(z_{\omega}\right)$. 
Proof. If the limit exists, it is equal to $\mathscr{H}^{\beta}\left(z_{\omega}\right)$ by the theorem on comparable net measures (see Chap. 2 of [23]). The problem that remains is the existence of the limit that is postponed to the Theorem 3.2 of the next section.

Remark. Notice that the estimate $Z_{\omega, k}(\beta)$ of the Hausdorff measure can be written as the partition function of a disordered system in the form explained in the Introduction. The Hausdorff measure of order $\beta$ of $z_{\omega}$ appears as the thermodynamic limit of $Z_{\omega, k}(\beta)$.

\section{Thermodynamics}

It is pointed out in the last section that the recursive construction of $z_{\omega}$ allows one to express the Hausdorff measure of this set as a statistical sum (partition function) of a disordered system. This analogy is not a new one. It has been already used for the Hausdorff measure of deterministic fractals [2].

The question that arises here is whether the disordered statistical systems that naturally appear in this random construction have any physical relevance. In fact, at the level $k$ there are $2^{k}$ terms appearing in the statistical sum and each such term is non-negative, hence it can be written as $\exp \left(-\beta \varepsilon_{s}\right)$, where $\varepsilon_{s}=-\log \lambda\left(K_{s}\right)$ is a random energy. Moreover, for $s, s^{\prime} \in M^{k}$ the corresponding energies are correlated and $E\left[\varepsilon_{s} \varepsilon_{s^{\prime}}\right]-E\left[\varepsilon_{s}\right] E\left[\varepsilon_{s^{\prime}}\right]=c\left[k-d\left(s, s^{\prime}\right)\right]$, where $d$ is the ultrametric distance defined for every $s, s^{\prime} \in M^{k}$ as

$$
d\left(s, s^{\prime}\right)=k-\max \left\{i:\left.s\right|_{i}=\left.s^{\prime}\right|_{i}\right\}
$$

Thus, the model studied here is reminiscent of the Generalised Random Energy Model (GREM) introduced in [9] and studied in [25]. There is nevertheless an important difference: contrary to Derrida's approach of the GREM, here the hamiltonian is bounded from below, guaranteeing thus the stability of the model. However, in view of the special construction used, the model must be viewed merely as a model providing some understanding of the real case.

This warning being given, I study here the thermodynamics of the model.

Lemma 3.1. Let $\phi(\beta)=E\left[\left(\lambda\left(K_{s \vee 1}\right)^{\beta}+\lambda\left(K_{s \vee 2}\right)^{\beta}\right) / \lambda\left(K_{s}\right)^{\beta}\right]$. Then,

i) $\phi$ is independent of $s \in M^{*}$,

ii) $\phi$ is strictly decreasing in $\beta$,

iii) $\phi(1 / 2)=1$.

Proof. i) For every $s \in M^{*}$, the Brownian bridge over $K_{s}$ and the rescaled bridges over $K_{s \vee 1}$ and $K_{s \vee 2}$ have the same pobability distribution. Pushing the argument back to the root of the tree, $\phi(\beta)$ is equal to $E\left(\lambda\left(K_{1}\right)^{\beta}+\lambda\left(K_{2}\right)^{\beta}\right)$.

ii) The decrease in $\beta$ follows immediately from the observation that for every $\omega \in \Omega$, $\lambda\left(K_{s \vee 1}(\omega)\right) / \lambda\left(K_{s}(\omega)\right) \leqq 1 / 2$.

iii) Using the explicit form for the bivariate probability distribution,

$$
\rho\left(\lambda\left(K_{1}(\omega)\right) \in d x, \lambda\left(K_{2}(\omega)\right) \in d y\right)=(2 \pi)^{-1} 1_{[0,1 / 2]^{2}}(x, y)\left[x y(1-x-y)^{3}\right]^{-1 / 2} d x d y,
$$

derived in the Appendix, it is shown by direct computation that $\phi(1 / 2)=1$. 
It is possible now to state the main result about the thermodynamic limit of the partition function.

Theorem 3.2. The sequence of random variables $Z_{k}(\beta)$ converges almost surely to a random variable $Z(\beta)$ such that

i) for $\beta>\beta_{c}=1 / 2, Z(\beta)=0$ almost surely,

ii) for $\beta<\beta_{c}=1 / 2, Z(\beta)=\infty$ almost surely and the limit $\lim Z_{k}(\beta) / \phi(\beta)^{k}$ is a.s. strictly positive.

Proof. i) Consider the $\sigma$-algebra $\mathscr{F}_{k}$ generated by the random variables

$$
X_{s, i}=\lambda\left(K_{\left.s\right|_{i+1}}\right) / \lambda\left(K_{\left.s\right|_{i}}\right)
$$

for $s \in M^{k}$ and $i=0, \ldots, k-1$. The sequence $\left\{\mathscr{F}_{k}, k \in \mathbf{N}\right\}$ is a filtration. Moreover, due to the rescaling of the Brownian bridge, the variables $X_{s, i}$ are i.i.d. Now, write

$$
Z_{k}(\beta)=\sum_{s \in M^{k}} \lambda\left(K_{s}\right)^{\beta}=\sum_{s \in M^{k}} \prod_{i=0}^{k-1} X_{s, i}^{\beta}
$$

and take the conditional expectations

$$
E\left(Z_{k}(\beta) \mid \mathscr{F}_{k-1}\right)=\sum_{s \in M^{k}} E\left(\frac{\lambda\left(K_{s}\right)^{\beta}}{\lambda\left(K_{s|s|-1}\right)^{\beta}}\right)_{i=0}^{k-2} X_{s, i}^{\beta}=\phi(\beta) Z_{k-1}(\beta) .
$$

Defining the sequence of random variables $W_{k}(\beta)=Z_{k}(\beta) / \phi(\beta)^{k}$, one sees immediately that $\left\{W_{k}, \mathscr{F}_{k}\right\}_{k \in \mathbf{N}}$ is a positive martingale, hence it converges to a random variable $W(\beta)$ with $0 \leqq W(\beta)<\infty$. The statement follows trivially because $\phi(\beta)<1$ for $\beta>1 / 2$.

ii) Denote by $M^{k}(\beta, \delta)$ the random set

$$
M^{k}(\beta, \delta)=\left\{s \in M^{k}: \lambda\left(K_{s}\right)^{\beta} \geqq \delta^{k}\right\}
$$

for $0 \leqq \delta \leqq 1$. It is easy to establish the inequalities

$$
\delta^{k} E\left(\operatorname{card} M^{k}(\beta, \delta)\right) \leqq \phi(\beta)^{k} \leqq k \sup _{0 \leqq \delta \leqq 1} \delta^{k-1} E\left(\operatorname{card} M^{k}(\beta, \delta)\right) .
$$

Therefore, if $\beta<\beta_{c}, \phi(\beta)>1$ and it is possible to find some integer $k$ and some $\delta \in[0,1]$ such that $\delta^{k} E\left(\operatorname{card} M^{k}(\beta, \delta)\right)>1$. Then using the Lemma A.3 of the Appendix and the fact that $z_{\omega} \neq \varnothing$, it follows that for $\beta<\beta_{c}, \rho\left(\left\{Z_{\infty}(\beta)=\infty\right\}\right)=1$. Now, by the standard theorem on the branching process, stating that either $W=0$ almost surely or $\{W>0\}=\{Z=\infty\}$ almost surely [1], we establish that $W>0$ a.s.

Using the identification of $Z(\beta)$ with $\mathscr{H}^{\beta}\left(z_{\omega}\right)$ as explained in the previous section one has the

Corollary 3.3. The Hausdorff dimension of the zero-level set of the Brownian bridge is a.s. $1 / 2$.

The previous theorem, showing that the partition function vanishes at low temperature, is the first step towards proving the existence of a phase transition. Actually, if there is a phase transition the partition function vanishes, by the Lee 
and Yang theorem. However, vanishing of the partition function is not a sufficient condition since models can be imagined where the partition function vanishes but which do not present any phase transition; it must be proved that there are in fact more than one state at low temperature. The existence of many states at low temperature in the thermodynamic limit is shown in the next section. Here, the definition of the state, adapted from [7], is given.

Definition 3.4. The "finite-volume" state is a random probability measure $\pi$ on the measurable space $([0,1], \mathscr{B}([0,1]))$ defined for every $A \in \mathscr{B}([0,1])$ by

$$
\pi_{\beta, N}(A)=Z_{N}(\beta)^{-1} \sum_{s \in M^{N}} \lambda\left(K_{s}(\omega) \cap A\right)^{\beta} .
$$

The problem as usual is the thermodynamic limit $(N \rightarrow \infty)$. As far as $N$ is finite $\pi_{\beta, N}$ is well defined (unique). In the limit $N \rightarrow \infty$ the probability measure $\pi_{\beta}$ remains well defined if $Z(\beta)>0$ (i.e. at high temperature) but it may fail to exist if $Z(\beta)=0$.

Instead of exploring the whole Borel $\sigma$-field $\mathscr{B}([0,1])$ it is enough to study $\pi_{\beta, N}\left(D_{s}\right)$ for every $s \in M^{k}$ with $N \geqq k$ and eventually take the limit $N \rightarrow \infty$. An important role in the intermediate technical steps is also played by the unnormalised random measure $\mu_{\beta, N}$ on $\mathscr{B}([0,1])$ defined for every $A \in \mathscr{B}([0,1])$ by

$$
\mu_{\beta, N}(A)=\sum_{s \in M^{N}} \lambda\left(K_{s}(\omega) \cap A\right)^{\beta} .
$$

\section{Study of the Free Energy and the States in the Thermodynamic Limit}

The finite-volume free energy is defined as usual to be the random variable

$$
f_{k}(\beta)=\frac{1}{k} \log Z_{k}(\beta)
$$

This section is devoted to the study of the $\operatorname{limits} \lim _{k \uparrow \infty} f_{k}(\beta)$ and "lim" $\pi_{k \uparrow \infty}$.

4.1. Self-Averaging and Unicity at High Temperature. It is generally believed [22] that when dealing with disordered systems, the annealed-i.e. $\lim _{k \uparrow \infty}(1 / k) \log E\left(Z_{k}(\beta)\right)$ and quenched -i.e. $\lim (1 / k) E\left(\log Z_{k}(\beta)\right)$-averages of the free energy coincide at high temperature. This phenomenon is known as the self-averaging property of the free energy. Here, an even stronger result is shown in the following

Proposition 4.1. (Strong self-averaging property). For $\beta<\beta_{c}, f(\beta)=\lim _{k \uparrow \infty} f_{k}(\beta)$ is almost surely a constant equal to the annealed mean.

Proof. The annealed average is $\log \phi(\beta)$ because $E\left(Z_{k}(\beta)\right)=E\left(Z_{k}(\beta) \mid \mathscr{F}_{0}\right)=\phi(\beta)^{k}$. Now, for $\beta<\beta_{c}$ the $\operatorname{limit} \lim Z_{k}(\beta) / \phi(\beta)^{k}=W(\beta)$ is almost surely $0<W(\beta)<\infty$ as shown in Theorem 3.2. Hence, $\lim _{k \uparrow \infty}(1 / k)\left(Z_{k}(\beta) / \phi(\beta)^{k}\right)=0$ a.s.

The other important, but immediate, result at high temperature is summarised in the following 
Proposition 4.2. (Unicity). For $\beta<\beta_{c}$, there is an almost surely unique random probability measure $\pi_{\beta}$ on $\mathscr{B}([0,1])$, obtained as limit of $\pi_{\beta, k}$ for $k \uparrow \infty$.

Proof. Fix $A$ to be a dyadic interval $D_{l}$ with $l \in M^{N}, N<k$ and write

$$
\pi_{\beta, k}(A)=\frac{\sum_{s \in M^{k}} \lambda\left(K_{s} \cap A\right)^{\beta} / \phi(\beta)^{k}}{Z_{k}(\beta) / \phi(\beta)^{k}} .
$$

Both the numerator and the denominator are positive martingales, therefore they converge respectively to the random variables $w(A)$ and $W$. By Theorem $3.2, W>0$ a.s. and by the positive martingale convergence Theorem, $0 \leqq w(A)<\infty$. Hence the ratio $w(A) / W$ is a well defined random variable $\pi_{\beta}(A) \leqq 1$ and $\pi_{\beta}([0,1])=1$. Thus, $\pi_{\beta}(\cdot)$, defined on the dyadic intervals, can be uniquely extended to a probability measure over $\mathscr{B}([0,1])$, for $\beta<\beta_{c}$. Hence, the $\operatorname{limit} \lim \pi_{\beta, k}$ exists and is a probability measure on $\mathscr{B}([0,1])$.

4.2. Existence of Many Limits and Lack of Self-Averaging at Low Temperature. The natural question whether the self-averaging fails at low temperature is answered in the following

Theorem 4.3. (Lack of self-averaging). For $\beta$ sufficiently larger than $\beta_{c}$,

Proof. Write

$$
\underset{k \uparrow \infty}{\limsup } \frac{1}{k} \log \frac{Z_{k}(\beta)}{\phi(\beta)^{k}}<0 \quad \text { a.s. }
$$

$$
W_{k}(\beta)=\frac{Z_{k}(\beta)}{\phi(\beta)^{k}}=\sum_{s \in M^{k}} \frac{\lambda\left(K_{s}\right)^{\beta}}{\phi(\beta)^{k}}=\sum_{s \in M^{k}} \prod_{i=0}^{k-1}\left(\frac{X_{s, i}^{\beta}}{\phi(\beta)}\right)
$$

where $X_{s, i}=\lambda\left(K_{\left.s\right|_{i+1}}\right) / \lambda\left(K_{\left.s\right|_{\imath}}\right)$. Therefore,

$$
\begin{aligned}
W_{k}(\beta) & =\sum_{s \in M^{k}} \exp \left[\beta \sum_{i=0}^{k-1} \log X_{s, i}-k \log \phi(\beta)\right] \\
& =\exp [k(\beta E(\log X))-\log \phi(\beta)] \sum_{s \in M^{k}} \exp \left[\beta \sum_{i=0}^{k-1} Y_{s, i}\right],
\end{aligned}
$$

where $Y_{s, i}=\log X_{s, i}-E\left(\log X_{s, i}\right)$. Notice that $X_{s, i}$ are i.i.d. and so are the quantities $Y_{s, i}$. Now, $E\left(Y_{s, i}\right)=0$ and $E\left(\left|Y_{s, i}\right|\right)<\infty$; by the law of large numbers $\lim k^{-1} \sum_{i=0}^{k-1} Y_{s, i}=0$ a.s. Consequently,

$$
\sum_{k=1}^{\infty} \rho\left(\left\{\left|Y_{k}\right|>\varepsilon k\right\}\right)<\infty
$$

for every $\varepsilon>0$, by Borel-Cantelli lemma. Write $\left|Y_{k}\right|=\left|\sum_{i=1}^{k} Y_{i}-\sum_{i=1}^{k-1} Y_{i}\right| \leqq\left|\sum_{i=1}^{k} Y_{i}\right|+$ $\left|\sum_{i=1}^{k-1} Y_{i}\right|$. Thus, $\left\{\left|\sum_{i=1}^{k} Y_{i}\right|>\varepsilon k / 2\right\} \subseteq\left\{\left|Y_{k}\right|>\varepsilon k\right\}$ and

$$
\sum_{k=1}^{\infty} \rho\left(\left\{\left|\sum_{i=1}^{k} Y_{i}\right|>\varepsilon k / 2\right\}\right)<\infty .
$$


Now, $\rho\left(\sum_{s \in M^{k}} \exp \left(\beta \sum Y_{i}\right)>2^{k} \exp (\beta \varepsilon k / 2)\right)<2^{k} \rho\left(\left|\sum Y_{i}\right|>\varepsilon k / 2\right)$. Choosing $\varepsilon>\log 2$ it is possible to render $\rho\left(\left|Y_{k}\right|>\varepsilon k\right)<2^{-k} k^{-2}$. By Borel-Cantelli it follows that the event $\left\{\exp \left(\beta \sum Y_{i}\right)>2^{k} \exp (\beta \varepsilon k / 2)\right.$ i.o. $\}$ is a null set. Otherwise stated, it happens that $\sum_{s \in M^{k}} \exp \left(\beta \sum Y_{i}\right) \leqq 2^{k} \exp (\beta \varepsilon k / 2)$ occurs infinitely often. Substituting, we get

$$
\limsup _{k \uparrow \infty} \frac{1}{k} \log W_{k}<E\left(\log X^{\beta}\right)-\log E\left(X^{\beta}\right)+\beta \log 2 / 2 .
$$

The proof is completed by noticing that $E\left(\log X^{\beta}\right)-\log E\left(X^{\beta}\right)+\beta \log 2 / 2$ can be made strictly negative for $\beta$ sufficiently large (larger than $\beta_{c}$ ).

The next important question that has to be answered is whether at low temperature there are many states or not. It will be shown that in general (i.e. if $A=\varnothing$ and $A \neq[0,1])$ the sequence $\left\{\pi_{\beta, k}(A), k \in \mathbf{N}\right\}$ admits at least two subsequences converging almost surely to different limits, if $\beta$ is sufficiently large.

Before stating the main result, two particular subsets of $M^{*}$ are introduced that can be easily visualised of one thinks of elements of $M^{*}$ in terms of branches of a dyadic tree. For every $s \in M^{*}$, the set $u m b(s) \equiv\left\{t \in M^{*}:\left.t\right|_{|s|}=s\right\}$ is called the umbra of $s$ and the set pen $(s) \equiv\left\{t \in M^{*}: \exists n\right.$, with $0<n \leqq|s|$ s.t. $\left.\forall i \leqq n,\left.s\right|_{i}=\left.t\right|_{i}\right\}$ is called the penumbra of $s$.

We need furthermore the extension of the Borel-Cantelli lemma to the case of non-independent events.

Lemma 4.4 (Conditional Borel-Cantelli lemma). Let $(\Omega, \mathscr{F}, \rho)$ be a probability space and $\left\{A_{n}, n \in \mathbf{N}\right\}$ be a sequence of events adapted to the filtration $\left\{\mathscr{F}_{n} \subset \mathscr{F}, n \in \mathbf{N}\right\}$, i.e. $A_{n}$ is $\mathscr{F}_{n}$ measurable. Then the sets $\left\{A_{n}\right.$, i.o. $\},\left\{\sum_{n=1}^{\infty} \rho\left(A_{n} \mid \mathscr{F}_{n-1}\right)=\infty\right\}$, and $\left\{\sum_{n=2}^{\infty} \rho\left(A_{n} \mid \mathscr{F}_{n-2}\right)=\infty\right\}$ are a.s. equal.

Proof. Apply Lemma A.1 of the Appendix to the bounded random variables $x_{n}=\mathbb{1}_{A_{n}}$.

A technical result is summarised in the following

Lemma 4.5. Let $(\Omega, \mathscr{F}, \rho)$ be a probability space and $\left\{X_{n}, n \in \mathbf{N}\right\}$ be a sequence of random variables $\Omega \rightarrow \mathbf{R}$ adapted to the filtration $\left\{\mathscr{F}_{n} \subset \mathscr{F}, n \in \mathbf{N}\right\}$. Fix some Borel set $A \in \mathscr{B}(\mathbf{R})$ and denote by $A_{n}=\left\{X_{n} \in A\right\}$. The sequence of random variables $\left\{Y_{n}, n \in \mathbf{N}\right\}$ defined by $Y_{n}=\sum_{i=1}^{n} \rho\left(A_{i+1} \mid \mathscr{F}_{i}\right)$ is a non-negative submartingale with respect to the filtration $\left\{\mathscr{F}_{n} \subset \mathscr{F}, n \in \mathbf{N}\right\}$.

Proof. $X_{n}$ being adapted, the events $A_{n}$ are obviously $\mathscr{F}_{n}$ measurable. Therefore, $Y_{n}$ are adapted to $\mathscr{F}_{n}$. Now,

$$
\begin{aligned}
E\left(Y_{n+1} \mid \mathscr{F}_{n}\right) & =\sum_{i=1}^{n} E\left(\rho\left(A_{i+1} \mid \mathscr{F}_{i}\right) \mid \mathscr{F}_{n}\right)+E\left(\rho\left(A_{n+2} \mid \mathscr{F}_{n+1}\right) \mid \mathscr{F}_{n}\right) \\
& =Y_{n}+E\left(\rho\left(A_{n+2} \mid \mathscr{F}_{n+1}\right) \mid \mathscr{F}_{n}\right) \geqq Y_{n} .
\end{aligned}
$$

The main result of this subsection is summarised in terms of the following 
Theorem 4.6 (Non-Unicity). Fix $A=[0,1 / 2[\subseteq K$. For every $\varepsilon \in] 0,1 / 4[$ there is a $\beta_{0}=\beta_{0}(\varepsilon)>\beta_{c}$ such that

$$
\left|\limsup _{k \uparrow \infty} \pi_{\beta, k}(A)-\underset{k \uparrow \infty}{\liminf } \pi_{\beta, k}(A)\right| \geqq \varepsilon
$$

for $\beta>\beta_{0}(\varepsilon)$.

Proof. Since the sequence of $\pi_{\beta, k}(A)$ is bounded, there is at least one converging subsequence. It will be shown that there are at least two subsequences converging to different limits. The proof uses essentially the upcrossing-downcrossing inequalities in the reverse way of the one used in the standard proof of the martingale convergence theorem.

Write

$$
\begin{aligned}
Z_{N}(\beta) & =\sum_{t \in M^{N}} \lambda\left(K_{t}\right)^{\beta} \\
& =\sum_{t \in M^{N} \cap \operatorname{umb}(A)} \lambda\left(K_{t}\right)^{\beta}+\sum_{\substack{t \in \operatorname{pen}(A) \backslash \operatorname{umb}^{N}(A) \\
t \in M^{N}}} \lambda\left(K_{t}\right)^{\beta}+\sum_{t \in M^{N} \backslash \operatorname{pen}(A)} \lambda\left(K_{t}\right)^{\beta},
\end{aligned}
$$

where $\operatorname{umb}(A)=\bigcup_{s: K_{s} \subset A} \operatorname{umb}(s)$ and $\operatorname{pen}(A)=\bigcup_{s: K_{s} \subset A} \operatorname{pen}(s)$. The particular choice of the set $A$ is such that pen $(A)=\operatorname{umb}(A)$ and the same holds for the set $A^{c}=K \backslash A$, hence the second term in the above sum vanishes and

$$
Z_{N}(\beta)=\mu_{\beta, N}(A)+\mu_{\beta, N}\left(A^{c}\right) .
$$

Choose $a=1 / 2-\varepsilon$. We show that $\pi_{\beta, k}(A)<a$ happens infinitely often. The difficulty here is that $\pi_{\beta, k-1}(A)$ and $\pi_{\beta, k}(A)$ are not independent random variables. Hence the standard Borel-Cantelli lemma is of no use. Consider therefore the sequence of events

$$
A_{k}=\left\{\mu_{\beta, k}(A)(1-a)<\mu_{\beta, k}\left(A^{c}\right) a\right\} .
$$

It is easy to see that $\left\{\pi_{\beta, k}(A)<a\right\}=A_{k}$ and moreover $A_{k} \in \mathscr{F}_{k}$. By Lemma 4.5, the sequence of random variables $Y_{k}=\sum_{n=1}^{k} \rho\left(A_{n} \mid \mathscr{F}_{n-1}\right)$ is a submartingale. By Doob's decomposition theorem (see [8] for instance), it can be written as $Y_{k}=M_{k}+I_{k}$, where $M_{k}$ is a martingale and $I_{k}$ is an increasing process given by $I_{k}=\rho\left(A_{k+1} \mid \mathscr{F}_{k-1}\right)$. The process $I_{k}$ being increasing, it is enough to show by explicit computation that for $\beta$ sufficiently large $\rho\left\{I_{2}>\theta\right\}=1$, for some positive $\theta$, since then $\sum \rho\left(A_{k+1} \mid \mathscr{F}_{k-1}\right)=\infty$ and by the conditional Borel-Cantelli Lemma 4.4 the event $A_{k}$ happens i.o

\section{The Critical Point}

The study of the critical point presents a particular interest because the probability measure corresponding to the thermodynamic limit can be explicitly constructed. Moreover, the unnormalised measure - related to the problem of self-intersections 
of random walks - was at the origin of the present study [17]. Namely, the unnormalised measure is proportional to the local time at zero.

In the Introduction, the notion of local time was given formally in terms of intuitive definitions. Here precise statements are made.

Definition 5.1 [15]. The occupation time for the Brownian bridge is the random measure $\Gamma_{t}(\cdot, \omega)$ on $\mathscr{B}(\mathbf{R})$ defined for every Borel set $A$ by

$$
\Gamma_{t}(A, \omega)=\int_{0}^{t} \mathbb{1}_{A}\left(B_{s}\right) d s=\lambda\left(\left\{s \in[0, t]: B_{s} \in A\right\}\right)
$$

for $0 \leqq t \leqq 1$

Definition 5.2. If the random measure $\Gamma_{t}(\cdot, \omega)$ is absolutely continuous with respect to the Lebesgue measure $\lambda$, the local time $L_{t}$ is defined as the Radon-Nikodym derivative $d \Gamma / d \lambda$.

Remark. The previous definition means that for every $A \in \mathscr{B}(\mathbf{R})$ and $0 \leqq t \leqq 1$,

$$
\Gamma_{t}(A, \omega)=\int_{A} d x L_{t}(x, \omega) .
$$

The main result of this section follows:

Theorem 5.3. There is a positive constant $c$ and a function $h$ given by the formula $h(y)=(y \log |\log y|)^{1 / 2}$ on $\mathbf{R}^{+}$for which

$$
\mathscr{H}^{(h)}\left(z_{\omega} \cap[0, t]\right)=c L_{t}(0, \omega)
$$

for $0 \leqq t \leqq 1$.

Before proceeding to the proof of this theorem some comments should clarify its significance. In the previous sections we used as $a$ priori measure on the intervals $[a, b], 0 \leqq a \leqq b \leqq 1$ the Lebesgue measure $\lambda([a, b])=b-a$. If instead of $\lambda$ we use some set function

$$
m([a, b])=(b-a) \log |\log (b-a)|
$$

( $m$ is not a measure) and construct the corresponding probability measure $\pi_{1 / 2, \omega}^{(m)}(\cdot)$ on $\mathscr{B}([0,1])$ by adapting the Definition 3.4 , the previous theorem states that every interval $[a, b] \subset[0,1]$ (i.e. every configuration) has a probability

$$
\pi_{1 / 2, \omega}^{(m)}([a, b])=\frac{L_{b}(0, \omega)-L_{a}(0, \omega)}{L_{1}(0, \omega)} .
$$

The interest of the previous formula stems from the fact that the local time can be computed by other methods $[24,18,29]$ thus it provides an explicit construction of the limit probability measure!

Definition 5.4 [5]. A process $Y_{t}$ is stable if its characteristic function $\chi(z ; t)=$ $E \exp \left(i z Y_{t}\right)$ can be written as

$$
\chi(z ; t)=\exp \left\{i \gamma z-c|z|^{\alpha}\left[1+i \beta \frac{z}{|z|} w(z, \alpha)\right]\right\},
$$


where $0<\alpha \leqq 2,|\beta| \leqq 1, c=c(t) \geqq 0$ and

$$
w(z, \alpha)=\left\{\begin{array}{lll}
\tan \pi \alpha / 2 & \text { if } \quad \alpha \neq 1 \\
2 \pi^{-1} \log |z| & \text { if } \quad \alpha=1
\end{array},\right.
$$

$\alpha$ is called the order of the process.

Lemma 5.5. The Brownian bridge $B_{t}$ over $[0,1]$ is a stable process of order 2 with $\beta=\gamma=0$ and $c(t)=t(1-t)$.

Proof. Let $\left\{X_{i}, i \in \mathbf{N}\right\}$ be a family of i.i.d. random variables uniformly distributed on $] 0,1]$ and form the family of random variables $Y_{t}^{n}=n^{-1 / 2} \sum_{i=1}^{n} 1_{\left\{X_{i} \leqq t\right\}}-n^{1 / 2} t$. $Y_{t}^{n}$ converges in law to $B_{t}$ [14]. Moreover the distribution function of $Y_{t}^{n}$ is infinitely divisible since $E \exp \left(i z Y_{t}^{n}\right)=\left[f_{n}(z)\right]^{n}$ with $f_{n}(z)=\left[t \exp \left(i z n^{-1 / 2}\right)+\right.$ $(1-t)] \exp \left(-i z n^{-1 / 2} t\right)$. Therefore the characteristic function of $B_{t}$ is $\chi(z ; t)=$ $\exp \left(-z^{2} t(1-t)\right)$.

Theorem 5.6. Suppose $X_{t}$ is a stable process of order $\alpha>1$ on the line with zero-level set $z_{\omega}$ and local time at zero $L_{t}$. Then there is a finite positive constant $c$ depending on the parameters of the process such that almost surely

$$
\mathscr{H}^{(h)}\left(z_{\omega} \cap[0, t]\right)=c L_{t}
$$

for all $t \geqq 0$, where $h(y)=y^{\theta}(\log |\log y|)^{1-\theta}$ and $\theta=1-1 / \alpha$.

Proof. See Theorem 1 of [26].

Proof of Theorem 5.3. The proof is an immediate consequence of Theorem 5.6 and Lemma 5.5.

\section{Conclusion}

It is shown in this paper that the model of disordered system considered here presents a phase transition at a critical temperature. Above the critical temperature the partition function does not vanish, the state is unique and the free energy has the self-averaging property. Below the critical temperature it is established that the partition function is zero; this is the starting point for showing that there are many states in the thermodynamic limit and that the free energy has not any self-averaging property. The methods used here are probabilistic.

The results proved here are in agreement with the results in [7] for a closely related model of multiplicative chaos. However, in spite of the formal similarities between the two models there are also important mathematical differences and the method used here to prove the multiplicity of the states at low temperature is different from the methods used in [7].

Is the method used here applicable in other disordered systems? Although the model considered is very special and cannot be transplanted as it stands to other situations, the probabilistic methods used to study it are very general and can be adapted.

It is worth recalling some genuine problems that still remain open. It is proved that there are at least two states at low temperature. How many states exist actually? 
It is believed that there is an infinite number of them but this is not proved. More fundamentally, what can be interpreted as a physical phase is an extremal thermodynamic state. Is there a procedure for selecting extremal states in the limit? Some very preliminary results seem to indicate that for this particular model there are only finitely many extremal states. Other closely related models should admit - by the same arguments - infinitely many states. Work in this direction is in progress. In case it is firmly established that there are infinitely many extremal states, here or in similar models, there is still a remaining open problem, namely the Choquet theory of infinitely many extremal states.

The thermodynamic state that arose at the critical point is well characterised; the corresponding probability measure can be written in terms of local time. It should be interesting to obtain explicit expressions for the probability measures at other temperatures.

In summarising, one can say that although some questions are rigorously answered in this work there are many important problems that still remain open.

\section{A. Appendix}

A.1. The Bivariate Distribution. In Sect. 2 the random times $\beta_{t}$ and $\gamma_{t}$ straddling $t$ were introduced for the Brownian bridge. These definitions are generalisations of the corresponding definitions for the Wiener process $W_{t}$; in fact it is in the context of Wiener processes where the random times $\beta_{t}^{\prime}$ and $\gamma_{t}^{\prime}$ straddling $t$ are initially introduced.

Now, use an equivalent definition of the Brownian bridge in terms of the Wiener process given by

$$
B_{t}=W_{t}-t W_{1}
$$

for $t \in[0,1]$. From the invariance of the probability distribution under the transformation $W_{t} \rightarrow t W_{1 / t}$ and the Proposition 3 of [6] stating that for $0<x<t<y$

$$
\operatorname{Prob}\left(\gamma_{t}^{\prime} \in d x, \beta_{t}^{\prime} \in d y\right)=\frac{d x d y}{2 \pi \sqrt{x(y-x)^{3}}},
$$

one obtains [19] for $0<x, y \leqq 1 / 2$,

$$
\rho\left(\gamma_{t} \in d x, 1-\beta_{t} \in d y\right)=\frac{d x d y}{2 \pi \sqrt{x y(1-x-y)^{3}}} .
$$

This last formula immediately leads to the form for the bivariate distribution of $\lambda\left(K_{1}\right)$ and $\lambda\left(K_{2}\right)$ claimed in the proof of Lemma 3.1 .

\section{A.2. A Useful Lemma}

Lemma A.1. Let $(\Omega, \mathscr{F}, \rho)$ be a probability space with a filtration $\left\{\mathscr{F}_{n} \subset \mathscr{F}, n \in \mathbf{N}\right\}$ on it, and $\left\{x_{n}\right\}$ a sequence of positive random variables adapted to $\mathscr{F}_{n}$. Let

$$
\begin{aligned}
& X_{n}^{(0)}=x_{0}+x_{1}+\cdots+x_{n}, \\
& X_{n}^{(1)}=x_{0}+E\left(x_{1} \mid \mathscr{F}_{0}\right)+E\left(x_{2} \mid \mathscr{F}_{1}\right)+\cdots+E\left(x_{n} \mid \mathscr{F}_{n-1}\right),
\end{aligned}
$$




$$
\begin{aligned}
X_{n}^{(2)}=x_{0} & +E\left(x_{1} \mid \mathscr{F}_{0}\right)+E\left(x_{2} \mid \mathscr{F}_{0}\right)+E\left(x_{3} \mid \mathscr{F}_{1}\right)+\cdots+E\left(x_{n} \mid \mathscr{F}_{n-2}\right), \\
\vdots & \\
X_{n}^{(k)}=x_{0} & +E\left(x_{1} \mid \mathscr{F}_{0}\right)+E\left(x_{2} \mid \mathscr{F}_{0}\right)+\cdots+E\left(x_{k} \mid \mathscr{F}_{0}\right) \\
& +E\left(x_{k+1} \mid \mathscr{F}_{1}\right)+\cdots+E\left(x_{n} \mid \mathscr{F}_{n-k}\right) .
\end{aligned}
$$

Denote by $X_{\infty}^{(j)}=\lim _{n \rightarrow \infty} X_{n}^{(j)}$ and by $A_{j}=\left\{X_{\infty}^{(j)}<\infty\right\}$. Then, $A_{k} \subseteq A_{k-1} \subseteq \cdots \subseteq A_{0}$ almost surely. If moreover $x_{n}$ are bounded uniformly (in $n$ ), these sets are almost surely equal.

Proof. $Y_{n-j+1}^{(j)}=X_{n}^{(j-1)}-X_{n}^{(j)}$ is a martingale and the random variable $T_{N}^{(j)}=$ $\inf \left\{n: X_{n}^{(j)}>N\right\}$ is a stopping time. The proof is completed by repeatedly applying $j$ times Theorem 46 of [8].

A.3. Branching Process Associated to the Random Recursive Construction. Given the random recursive construction on $M^{*}$ define for every $\delta$ with $0<\delta<1 / 2$ the random set

$$
M_{\delta}^{k}=\left\{s \in M^{k}: X_{\left.s\right|_{\imath}}>\delta, \forall i=1, \ldots, k\right\}
$$

and consider the limit $M_{\delta}^{*}=\bigcup_{k=0}^{\infty} M_{\delta}^{k}$. The random graph $M_{\delta}^{*}$ is isomorphic to the tree associated to an ordinary branching process having as progeny a random variable with the same distribution as the random variable $N=\operatorname{card}\left\{j \in M: X_{(j)}>\delta\right\}$. Obviously $N$ takes on the discrete values 0,1 , and $2=$ card $M$. Now the following is known from the standard theory of branching processes [1]:

Theorem A.2. If $E(N)>1$, there is a strictly positive probability for the branching process not to become extinct.

This theorem is used in [11] to prove a result about flows on random networks. Rephrased in the present case this result can be stated in the form of the following

Lemma A.3. If in a random recursive construction there is a $\delta$ with $0<\delta \leqq 1$ and $a$ positive integer $k$, such that

$$
\delta^{k} E\left(\operatorname{card}\left\{s \in M^{k}: \prod_{i=1}^{k} X_{\left.s\right|_{i}}>\delta^{k}\right\}\right)>1,
$$

then the corresponding branching process $M_{\delta}^{*}$ has a strictly positive probability not to become extinct.

Acknowledgments. I should like to thank F. Koukiou for numerous useful discussions and for communicating to me the results in $[16,7]$ prior to their publication.

\section{References}

1. Asmussen, S., Hering, H.: Branching processes. Basel: Birkhäuser 1983

2. Badii, R., Politi, A.: Statistical description of chaotic attractors. J. Stat. Phys. 40, 725-750 (1985)

3. Besicovitch, A. S.: On the fundamental geometrical properties of linearly measurable plane sets, I, II, and III. Math. Ann. 98, 422-464 (1927); 115, 296-329 (1938); 116, 349-357 (1939) 
4. Bricmont, J., Kupiainen, A.-J.: Phase transition in the $3 \mathrm{~d}$ Random Field Ising Model. Commun. Math. Phys. 116, 539-572 (1988)

5. Chow, Y. S., Teicher, H.: Probability theory. Berlin, Heidelberg, New York: Springer 1978

6. Chung, K. L.: Excursions in Brownian motion. Arkiv Math. 14, 155-177 (1978)

7. Collet, P., Koukiou, F.: Thermodynamics of the multiplicative chaos. Preprint Université de Lausanne (1989)

8. Dellacherie, C., Meyer, P.-A.: Probabilitiés et potentiel, vol. 2. Théorie des martingales. Paris: Hermann 1980

9. Derrida, B.: A generalization of the Random Energy Model which includes correlations between energies. J. Physique-Lett. 46, L401-L407 (1985)

10. Falconer, K. J.: The geometry of fractal sets, Cambridge Tracts in Mathematics vol. 85. Cambridge: Cambridge University Press 1985

11. Falconer, K. J.: Random fractals. Math. Proc. Camb. Phil. Soc. 100, 559-582 (1986)

12. Fröhlich, J., Zegarliński, B.: Spin glasses and other lattice systems with long range interactions. Commun. Math. Phys. 170, 665-688 (1989)

13. Hausdorff, F.: Dimension und äußeres Maß. Math. Ann. 79, 157-179 (1919)

14. Jacod, J., Shiryaev, A. N.: Limit theorems for stochastic processes. Grundlehren der mathematischen Wissenschaften vol. 288. Berlin, Heidelberg, New York: Springer 1987

15. Karatzas, I., Shreve, S. E.: Brownian motion and stochastic calculus. Berlin, Heidelberg, New York: Springer 1987

16. Koukiou, F.: A random covering interpretation of the Random Energy Model. Preprint Université de Lausanne (1988)

17. Koukiou, F., Pasche, J., Petritis, D.: The Hausdorff dimension of the two-dimensional Edwards' random walk. J. Phys. A: Math. Gen. (1988)

18. Le Gall, J.-F.: Sur les temps locaux d'intersection du mouvement Brownien et la méthode de renormalisation de Varadhan, in Séminaire de Probabilités XIX. Lecture Notes in Mathematics vol. 1123, pp. 314-331. Berlin, Heidelberg, New York: Springer 1986

19. Graf, S., Mauldin, R. D., Williams, S. C.: The exact Hausdorff dimension in random recursive constructions. Mem. A.M.S. 71. Number 381 (1988)

20. Lévy, P.: Processus stochastiques et mouvement Brownien. Paris: Gauthier-Villars 1948

21. Mauldin, R. D., Williams, S. C.: Random recursive constructions: Asymptotic, geometric, and topological properties. Trans. Am. Math. Soc. 295, 325-346 (1986)

22. Mézard, M., Parisi, G., Virasoro, M. A.: Spin glass theory and beyond. Singapore: World Scientific 1988

23. Rogers, C. A.: Hausdorff measures. Cambridge: Cambridge University Press 1970

24. Rosen, J.: A representation for the intersection local time of Brownian paths in space. Ann. Probab. 13, 145-153 (1985)

25. Ruelle, D.: A mathematical reformulation of Derrida's REM and GREM. Commun. Math. Phys. 108, 225-239 (1987)

26. Taylor, S. J., Wendell, J. G.: The exact Hausdorff measure of the zero set of stable processes. Z. Wahrscheinlichkeitstheorie Verw. Geb. 6, 170-180 (1966)

27. Varadhan, S. R. S.: Appendix to the course by K. Symanzik. In: Local quantum theory. Jost, R. (ed.) New York: Academic Press 1969

28. Westwater, J.: On Edwards' model for long polymer chains. Commun. Math. Phys. 72, 131-173 (1980); 79, 53-73 (1981); 84, 459-470 (1982)

29. Yor, M.: Renormalisation et convergence en loi pour les temps locaux d'intersection du mouvement brownien dans $\mathbf{R}^{3}$. In: Séminaire de Probabilités XIX. Lecture Notes Mathematics vol. 1123, pp. 350-365. Berlin, Heidelberg, New York: Springer 1986

Communicated by J. Fröhlich

Received January 11, 1989; in revised form May 2, 1989 
